\title{
Perforaciones post colangiopancreatografía endoscópica retrógrada (CPRE). Manejo quirúrgico
}

\section{Perforations following endoscopic retrograde cholangiopancreatography (ERCP)}

doi.org/10.23938/ASSN.0014

\author{
J. Mateo Retuerta, C. Chaveli Díaz, A. Goikoetxea Urdiain, B. Sainz Villacampa, \\ M.J. Sara Ongay, J.J. Íñigo Noain
}

\section{RESUMEN}

La colangiografía endoscópica retrógrada (CPRE) asociada a esfinterotomía es un procedimiento útil ampliamente utilizado en casos de coledocolitiasis y obstrucción de la vía biliar. A pesar de tratarse de una prueba segura, existe el riesgo de complicaciones tales como la pancreatitis, la hemorragia o la perforación.

La perforación duodenal post CPRE es un hecho poco común pero de graves consecuencias de no ser tratado precozmente. El mecanismo de lesión, el lugar y extensión de la perforación así como la clínica y los hallazgos radiográficos guiarán el manejo del paciente, que debe ser individualizado y en constante reevaluación.

Su mortalidad se relaciona de manera estrecha con el retraso diagnóstico y terapéutico. En aquellos en los que se decida una actitud conservadora, se mantendrá una vigilancia estrecha ante la posibilidad de empeoramiento clínico y cambio en su manejo.

Palabras clave: Perforación intestinal, Perforación duodenal, Colangiopancreatografía, Endoscopia retrógrada.

\begin{abstract}
Endoscopic retrograde cholangiopancreatography (ERCP) associated with sphincterotomy is a useful procedure that is widely used in cases of choledocholithiasis and bile duct obstruction. In spite of being a safe test, there is a risk of complications like pancreatitis, haemorrhaging or perforation.

Post-ERCP duodenal perforation is a rare event but has serious consequences if it is not treated early. The lesion mechanism, the place and extension of the perforation, as well as the clinical picture and radiographic findings will guide patient management, which must be individualised and constantly reevaluated.

Its mortality is closely related to diagnostic and therapeutic delay. In those cases where a conservative attitude is adopted, close vigilance will be maintained facing the possibility of a worsening of the clinical picture and a change in its management.
\end{abstract}

Keywords: Intestinal perforation. Duodenal perforation. Cholangiopancreatography. Retrograde endoscopy.
Área de Cirugía General. Complejo Hospitalario de Navarra. Pamplona. España.

Recepción: 25/10/2016

Aceptación provisional: 22/12/2016

Aceptación definitiva: 25/01/2017

\section{Correspondencia:}

Janire Mateo Retuerta

Área de Cirugía General

Complejo Hospitalario de Navarra

Irunlarrea, 3

31008 Pamplona

E-mail: janiremateo@gmail.com 


\section{INTRODUCCIÓN}

La colangiografía endoscópica retrógrada es un procedimiento diagnóstico y terapéutico útil para la solución de problemas pancreáticos y biliares ${ }^{1-10}$. Asociado a la esfinterotomía, puede utilizarse para la extracción de cálculos, drenaje del árbol biliar y colocación de prótesis ${ }^{9,10}$. A pesar de ser una prueba segura, existe el riesgo de complicaciones tales como la pancreatitis, la perforación o la hemorragia ${ }^{1-4,-9,11,12}$. La incidencia de complicaciones oscila entre el $5,4 \%$ y el $23,9 \%$ y la mortalidad del 0,1 al $1 \%$ 1,4-6,8,9,11 . La incidencia de perforación oscila entre el 1 y el $2 \%^{3-6,11,12}$ y su mortalidad asciende al $16-18 \%$ según las series descritas ${ }^{10}$. De cualquier forma, la mortalidad se relaciona con el retraso en el diagnóstico y el tratamiento, que deriva en sepsis y fallo multiorgánico. Tradicionalmente, tanto las perforaciones traumáticas como las no traumáticas han sido subsidiarias de tratamiento quirúrgi$\mathrm{co}^{5,6,9,11,12}$. Este enfoque ha cambiado en los últimos años, convirtiéndose en un manejo más selectivo e individualizado según las características del paciente ${ }^{5,9}$.

Dada la baja incidencia de complicaciones, no existe un consenso sobre el manejo para la decisión del tratamiento quirúrgico frente al conservador ${ }^{9,12}$ (Diagrama). Las graves consecuencias de la perforación post-CPRE obligan a un mejor entendimiento de los factores predisponentes y a la creación de una estrategia apropiada.

En un periodo de 22 meses (desde enero de 2015 a octubre de 2016), nuestro servicio de Cirugía fue consultado para la valoración quirúrgica de 12 perforaciones post-CPRE, de manejos diversos y con buena respuesta a los tratamientos instaurados. Dado el número creciente de estos procedimientos y el consecuente aumento de las complicaciones, creemos razonable presentar varios casos tipo como ejemplo de los posibles manejos terapéuticos existentes.

\section{CASOS CLÍNICOS}

\section{Caso 1}

Varón de 82 años que ingresa para realización de CPRE por un cuadro de colangitis aguda. Tras la extracción de cálculos y esfinterotomía laboriosa se objetiva enfisema subcutáneo en tórax, cuello y cara en ausencia de distensión abdominal. Presenta datos de acidosis metabólica y se mantiene afebril y hemodinámicamente estable.

La TAC demuestra neumomediastino, neumopericardio, neumotórax bilateral y un defecto

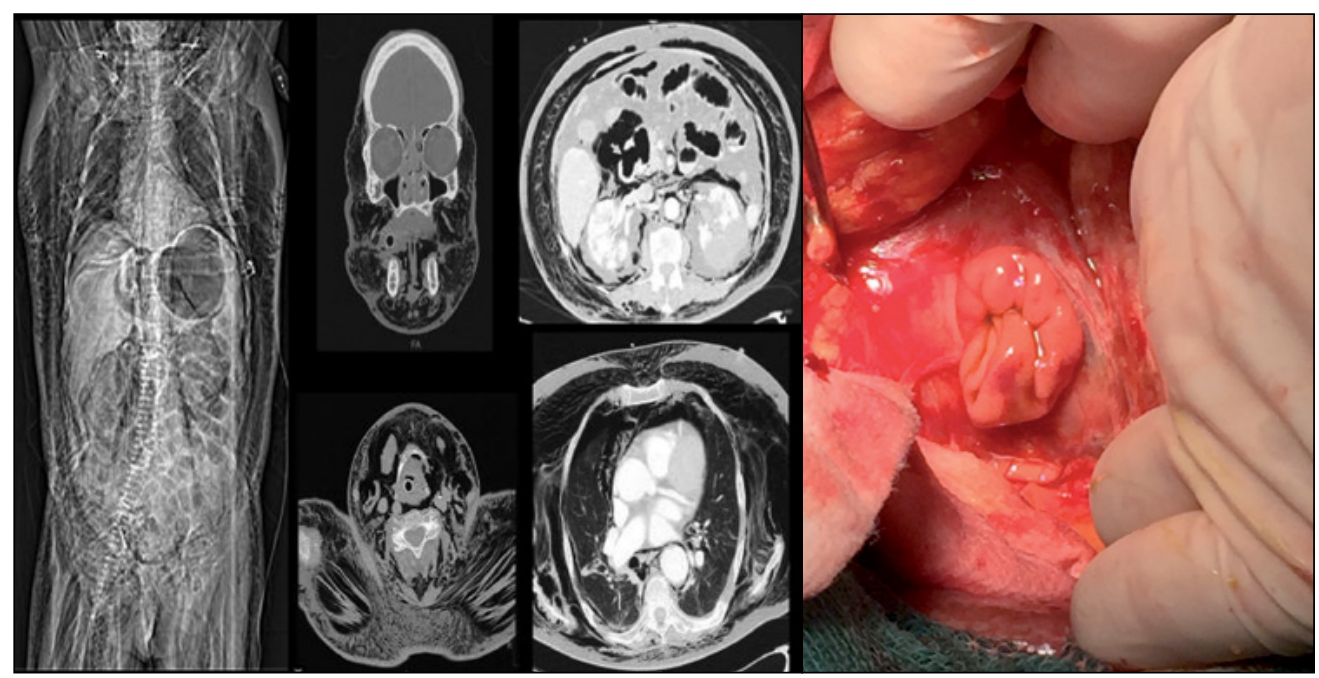

Figura 1.1: TC craneo-torácico-abdomino-pélvico. Neumomediastino, neumopericardio, neumotórax bilateral y perforación de 1,5 cm en cara anterolateral de segunda porción duodenal. Neumoperitoneo y retroneumoperitoneo. Voluminoso enfisema subcutáneo desde zona craneofacial y cervical hasta raíz de muslos y escroto. Figura 1.2: imagen intraoperatoria. Perforación duodenal con eversión mucosa. 


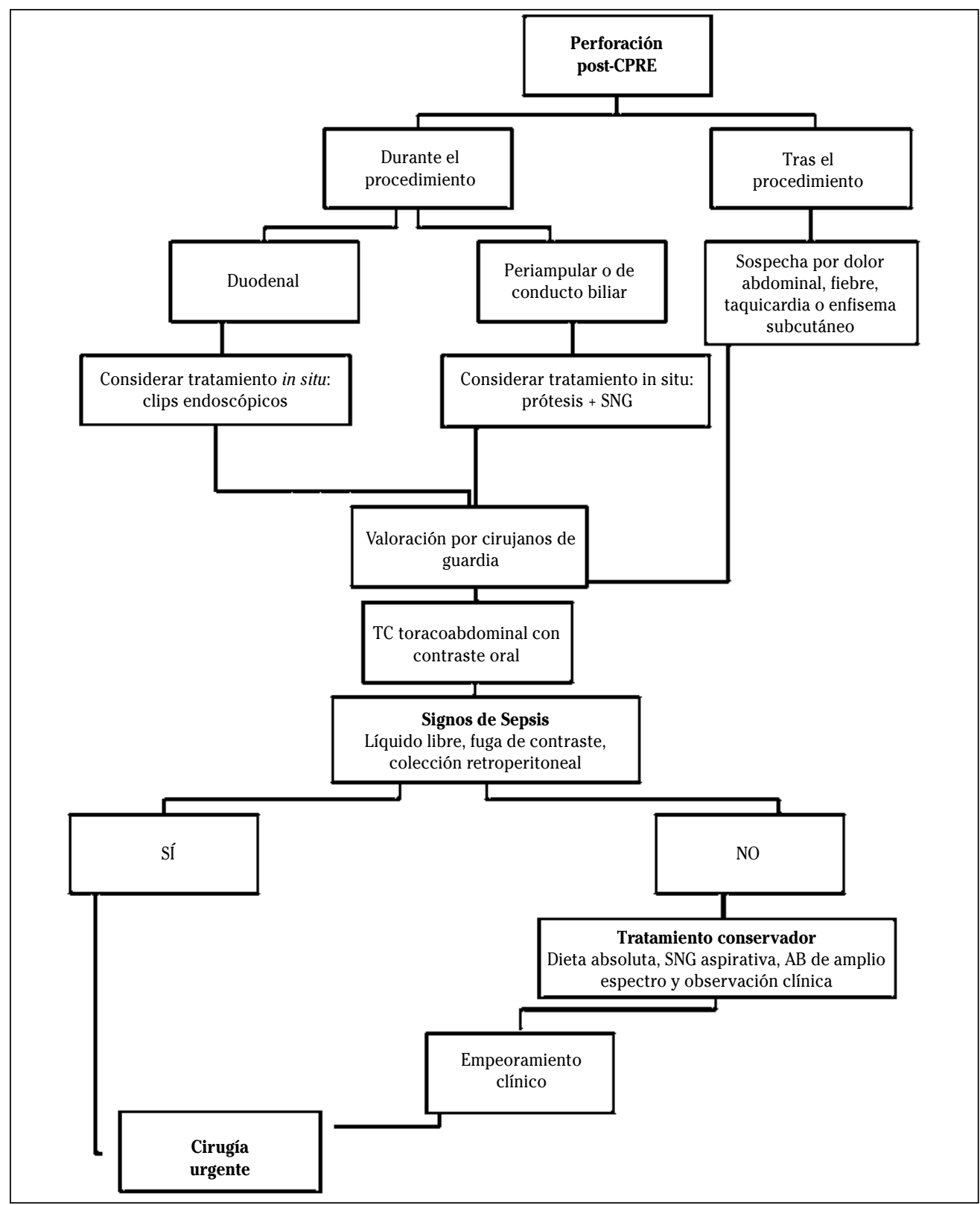

Diagrama. Esquema conceptual; manejo de la perforación post-CPRE.

en cara anterolateral de la segunda porción duodenal, frente a la papila, que condiciona neumoperitoneo y retroneumoperitoneo, así como un voluminoso enfisema subcutáneo que se extiende desde la zona craneofacial y cervical hasta la raíz de muslos y escroto (Fig. 1.1).

Ante los hallazgos presentes se decide la intervención quirúrgica urgente. Tras realizar la maniobra de Kocher se identifica una perforación en cara anterolateral de segunda porción duodenal de 1,5 cm con eversión mucosa (Figs. 1 y 2). Se realiza un cierre primario de la perforación y una colecistomía reglada, siendo dado de alta al $18^{\circ}$ día postoperatorio tras diez días de estancia en la Unidad de Cuidados intensivos con una evolución satisfactoria. 


\section{Caso 2}

Mujer de 70 años con diagnóstico de colelitiasis y coledocolitiasis sintomática confirmada mediante colangio-resonancia que ingresa para la realización de CPRE. La prueba resulta dificultosa por la presencia de un divertículo duodenal. Se realiza esfinterotomía y tras varios intentos se asciende en una dirección teórica hacia el Wirsung, hasta que se sospecha la existencia de falsa vía y perforación retroperitoneal por extravasación de contraste.

En la TC se objetiva un gran neumomediastino (Fig. 2.1), neumoperitoneo y retroneumoperitoneo

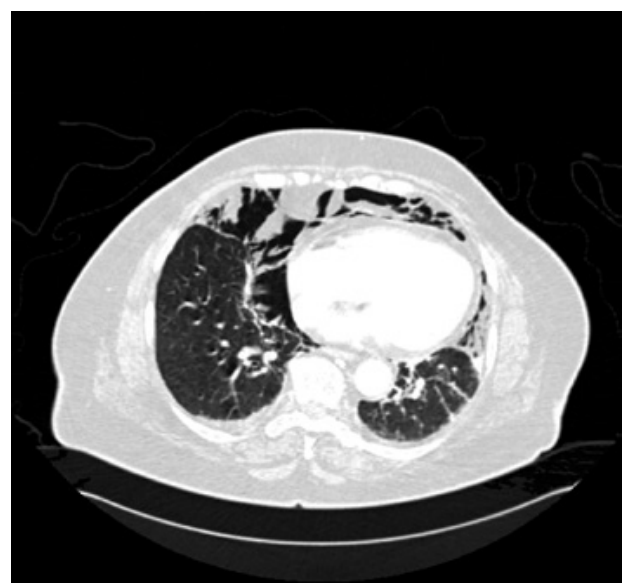

Figura 2.1: TC torácico. Neumomediastino.
(Fig. 2.2) con difusión a pelvis y conducto inguinal, sin llegar a localizar el lugar de la perforación. En las horas posteriores la clínica de la paciente empeora y evoluciona a una exploración con signos de irritación peritoneal por lo que se decide la intervención quirúrgica. Tras la maniobra de Kocher y la realización de una gastroscopia intraoperatoria, no se logra identificar el lugar de la perforación, por lo que se realiza una colecistectomía coledocotomía para la extracción de cálculos con colangiografía trans-Kehr posterior normal.

Tras una evolución satisfactoria que precisó estancia en la UCI, es dada de alta al $15^{\circ}$ día postoperatorio.

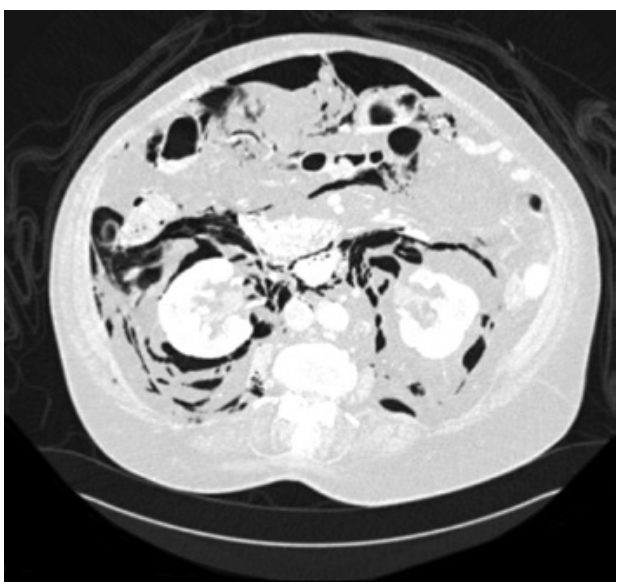

Figura 2.2: TC abdomino-pélvico. Neumoperitoneo y retroneumoperitoneo

consiste en nutrición parenteral y tratamiento antibiótico empírico, siendo dada de alta tras adecuada tolerancia oral progresiva.

\section{Caso 4}

Varón de 30 años con antecedentes de cólicos biliares de repetición que acude al servicio de Urgencias por un nuevo episodio de dolor abdominal con escalofríos, coluria, acolia e ictericia conjuntival. Presenta hiperbilirrubinemia y colelitiasis en la ecografía abdominal. Ingresa para la realización de una CPRE, practicándose una esfinterotomía laboriosa durante la cual el endoscopista transmite la sensación de perforación peripapilar. Dada la estabilidad hemodinámica del paciente, se decide la colocación de una prótesis metálica biliar que cubre el defecto. 
La TC abdominal posterior refleja retroneumoperitoneo peripancreático en hilio hepático y espacio perirrenal derecho. Dado el buen estado clínico del paciente y la ausencia de síntomas de alarma, se decide tratamiento conservador, siendo dado de alta en 10 días con una evolución satisfactoria.

\section{DISCUSIÓN}

La CPRE es un procedimiento terapéutico predominante en nuestro tiempo pero no está exenta de riesgos. Entre sus posibles complicaciones se encuentra la perforación duodenal, que a pesar de infrecuente puede tener graves consecuencias de no ser tratada precozmente. Sus factores predisponentes incluyen las relacionadas con el paciente y con la técnica (Tabla 1) $4,5,9,11,12$.
Las perforaciones relacionadas con la CPREse clasifican en tres grupos: el grupo I o perforación por guía de alambre, el grupo II o perforación periampular y el grupo III o perforación duodenal lejos de la papila $^{8}$. La más frecuentemente empleada es la clasificación propuesta por Stapfer y col${ }^{6}$ basada en el mecanismo, localización anatómica y severidad, en orden descendente de gravedad (Tabla 2).

Las perforaciones más frecuentes son las derivadas de la esfinterotomía (tipo II) y de la instrumentalización (tipo III) conformando entre ambas el $80 \%$ de las perforaciones ${ }^{8,9,11}$ y la que ocurre con menos frecuencia es la tipo I y aquella relacionada con la alteración anatómica gastrointestinal $^{6,13}$.

El diagnóstico y tratamiento precoz de la perforación duodenal tras la CPRE son la

Tabla 1. Factores de riesgo relacionados con la perforación tras colangiografía endoscópica retrógrada.

\begin{tabular}{|c|c|}
\hline & Factores de riesgo \\
\hline \multirow{6}{*}{$\begin{array}{c}\text { Relacionados } \\
\text { con el } \\
\text { paciente }\end{array}$} & Sospecha de disfunción del esfínter de Oddi \\
\hline & Sexo femenino \\
\hline & Edad avanzada \\
\hline & Niveles normales de bilirrubina \\
\hline & Historia previa de pancreatitis post-CPRE \\
\hline & Distorsión anatómica como situs inversus o gastrectomía por Billroth II \\
\hline \multirow{6}{*}{$\begin{array}{l}\text { Relacionados } \\
\text { con la técnica }\end{array}$} & Canulación dificultosa \\
\hline & Inyección de contraste en conducto pancreático \\
\hline & Procedimiento de larga duración \\
\hline & $\begin{array}{l}\text { Esfinterotomía y técnica precorte (sobre todo en incisiones más allá del sector entre } 11 \text { y } 1 \\
\text { horas) }\end{array}$ \\
\hline & Dilatación del esfínter mediante balón \\
\hline & Endoscopista poco experimentado \\
\hline
\end{tabular}

Tabla 2. Clasificación de perforaciones postCPRE según Stapfer.

\begin{tabular}{cl}
\hline & \multicolumn{1}{c}{ Perforación postCPRE según Stapfer } \\
\hline TIPO I & Pared lateral o medial duodenal \\
\hline TIPO II & Lesión periampolla de Váter \\
\hline TIPO III & Lesiones de conducto biliar distal relacionadas con instrumentación aro/canasta \\
\hline TIPO IV & Únicamente aire retroperitoneal (no se considera una perforación real) \\
\hline
\end{tabular}


base para un buen resultado ${ }^{5,6,9,10,14}$. Es una complicación de difícil diagnóstico debido a la sedación durante el procedimiento ${ }^{2,9}$. Los signos y síntomas sospechosos son el dolor epigástrico, la lumbalgia intensa, la irritación peritoneal, el enfisema subcutáneo, la taquicardia y la fiebre (estos dos últimos más tardíos) $)^{5-9,11,12}$.

En pacientes con dolor y fiebre tras el procedimiento se instaurará un tratamiento precoz a base de fluidos y antibioterapia empírica intravenosa. La radiografía de abdomen puede revelar aire libre intraperitoneal, pero el método más sensible y específico para su diagnóstico es la TC abdomino-pélvica con contraste oral ${ }^{5,12,15}$, que demuestra de forma efectiva aire retro e intraperitoneal con o sin extravasación de contraste oral ${ }^{4,5,9,11}$. En general, el aire libre intraperitoneal implica una fuga no contenida que suele requerir intervención quirúrgica, mientras que el aire retroperitoneal aislado es sugestivo de perforación en el lugar de la esfinterotomía ${ }^{15}$. De cualquier forma, la cantidad de aire libre no se correlaciona con el tamaño del defecto, sino con la cantidad de aire insuflado durante el procedimiento ${ }^{9}$.

Tras la resucitación y el diagnóstico precoz debe determinarse si el paciente va a requerir una intervención quirúrgica o se beneficiará de un manejo conservador. Debe tenerse en cuenta el mecanismo de lesión, el lugar y la extensión de la perforación así como la clínica y los hallazgos radiográficos $^{3,5,6,11,12}$ puesto que en las últimas décadas el manejo terapéutico se ha acercado más hacia un enfoque selectivo en cada caso ${ }^{4,9}$. Los objetivos son el control de la sepsis, mediante el drenaje de colecciones y del sistema biliar mediante la extracción de cálculos o material intraductal, y la reparación de la perforación.

La lesión tipo I (perforación duodenal) suele requerir una intervención quirúrgica. La técnica óptima consiste en el cierre primario del defecto y exclusión duodenal mediante gastroyeyunostomía y exclusión pilórica $^{4,6,9,13}$, aunque el cierre primario y desbridamiento de tejidos es suficiente, con similares resultados, en caso de diagnóstico y manejo precoz que aseguren una contaminación peritoneal mínima ${ }^{8,9,12}$. La indicación quirúrgica incluye además la fuga de contraste masivo, la perforación por CPRE con coledocolitiasis documentada, la retención de material (como la cesta de Dormia), el enfisema subcutáneo masivo o el fallo del tratamiento conservador $^{5,6,11}$. Otros factores que predisponen al tratamiento quirúrgico según el análisis multivariante de Fatima y col $^{16}$ y Knudson y $\operatorname{col}^{17}$ son la edad, la comorbilidad, los pacientes con perforación duodenal y una técnica endoscópica dificultosa.

Las perforaciones tipo II y III por instrumentación endoscópica causan lesiones más pequeñas y menos contaminación intraabdominal, y responden mejor a un manejo conservador ${ }^{5-7,9,10,15,18}$. El manejo conservador incluye dieta absoluta, sonda nasogástrica, antibióticos de amplio espectro y reevaluación continua ${ }^{4,5,8,9,12}$. En perforaciones tipo II y III este manejo tiene una evolución satisfactoria entre el 50 y el $90 \%$ de los $\operatorname{casos}^{8,9}$. La indicación quirúrgica en este tipo de perforaciones incluye el fracaso de tratamiento conservador, la presencia de líquido libre, colecciones retroperitoneales extensas y signos de fuga activos o de irritación peritoneal ${ }^{3,5,9,11,13}$.

El objetivo continúa siendo la distinción de los pacientes que puedan ser tratados de manera conservadora de los que necesiten cirugía urgente ${ }^{9}$. Entre otros, el retraso en el diagnóstico o en el tratamiento, el rescate quirúrgico tras el fracaso del manejo conservador, la reintervención quirúrgica y la edad avanzada son pronósticos de mala evolución ${ }^{5,6,8,9,13}$. Pese a todo, los resultados en los últimos años han mejorado de manera significativa gracias a la detección precoz y las estrategias de manejo apropiadas $^{9}$.

En conclusión, la perforación duodenal post CPRE es un hecho poco común pero de graves consecuencias si no es tratado de forma precoz. Es crucial el tiempo transcurrido entre la perforación y la valoración quirúrgica, puesto que un retraso en el tratamiento incrementa de forma significativa la mortalidad.

En general los pacientes con perforaciones tipo I necesitan de cirugía de mane- 
ra invariable y las tipo II y III pueden ser manejadas mediante tratamiento conservador. En cualquier caso todos requerirán observación y reevaluación cercana para confirmar el progreso satisfactorio del cuadro.

\section{BIBLIOGRAFÍA}

1. Yilmaz B, Roach EC, Koklu S, Aydin O, Unlu O, AlPER KILIC Y. Air leak syndrome after endoscopic retrograde cholangiopancreatography: A rare and fatal complication. World $\mathrm{J}$ Gastroenterol 2015; 21: 4770-4772.

2. García-Cano J, González JA, Morillas J, Pérez A. Complicaciones de la colangiopancreatografía retrógrada endoscópica: Estudio en una unidad pequeña de CPRE. Rev Esp Enferm Dig 2004; 96: 163-173.

3. Ozgonul A, Cece H, Sogut O, Demir D, Kurkcuoglus IC. Pneumoperitoneum, pneumoretroperitoneum and bilateral pneumothorax caused by ERCP. J Pak Med Assoc 2010; 60: 60-61.

4. Palomeque A, González C, Pérez D, Jiménez JA. Tratamiento conservador de la perforación duodenal tras realización de colangiopancreatografía endoscópica terapéutica. Gastroenterol Hepatol 2015; 38: 285-286.

5. LEE SM, Сно KB. Value of temporary stents for the management of perivaterian perforation during endoscopic retrograde cholangiopancreatography. World J Clin Cases 2014; 2: 689-697.

6. Stapfer M, Rick R, Stain CS, Katkhouda N, Parekh $\mathrm{D}$, JABBour $\mathrm{N}$ et al. Management of duodenal perforation after endoscopic retrograde cholangiopancreatography and sphincterotomy. Ann. Surgery 2000; 232: 191-198.

7. Brueck M, Bandorski D, Rauber K, LotTerer E. Pneumoretroperitoneum and bilateral pneumothorax after endoscopic biliary sphincterotomy. Dtsch Med Wochenschr 2010; 135: 853-856.

8. Howard TJ, Tan T, Lehman GA, Sherman S, MaDURA JA, Fogel E et al. Classification and management of perforations complicating en- doscopic sphincterotomy. Surgery 1999; 126 : 658-663.

9. MaCHADO NO. Management of duodenal perforation post-endoscopic retrograde cholangiopancreatography. When and whom to operate and what factors determine the outcome? A review article. JOP 2012; 13: 18-25.

10. Makn A, Chebbi F, Ben Safta Z. Pneumoretroperitoneum, bilateral pneumothorax and emphysema following endoscopic biliary sphincterotomy. Acta Chir Belg 2012; 112: 307-309.

11. Сно KB. The management of endoscopic retrograde cholangiopancreatography-related duodenal perforation. Clin Endosc 2014; 47: 341-345.

12. Armas MD, Ojeda V, Roque C, Cabrera JC, Mathías MP, Ceballos D et al. Perforaciones duodenales tras colangiopancreatografía retrógrada endoscópica. Cir Esp 2015; 93: 403410.

13. Turner RC, StefFen CM, Boyd P. Endoscopic duodenal perforation: surgical strategies in a regional centre. World J Emerg Surg 2014; 9: 11.

14. Völgyi Z, Szenes M, Gasztony B. Types and management of perforations occurring during endoscopic retrograde cholangiopancreatography. Orv Hetil 2014; 155: 248-254.

15. Vowinkel T, SENNinger N. Retroperitoneal emphysema after endoscopic retrograde cholangiopancreatography. Chirurg 2015; 86: 462-467.

16. Fatima J, Baron TH, Topazian MD, Houdhton SG, IGBAL CW, OTt BJ et al. Pancreaticobiliary and duodenal perforations after periampullary endoscopic procedures: Diagnosis and management. Arch Surg 2007: 142: 448-454.

17. Knudson K, Raeburn CD, McIntyre RC JR, ShaH RJ, Chen YK, Brown WR et al. Management of duodenal and pancreaticobiliary perforations associated with periampullary endoscopic procedures. Am J Surg 2008; 196: 975981.

18. John AK, Thandassery RB, Alam SH, Al KaABI SR. Extensive cervical, thoracic, and abdominal wall emphysema and pneumomediastinum following ERCP: a rare scenario. Ann Gastroenterol 2014; 27: 438-439. 
